\title{
CONVEXITY OF INTEGRAL MEANS OF SUBHARMONIC FUNCTIONS
}

\author{
JANG-MEI G. WU ${ }^{1}$
}

\begin{abstract}
We study the convexity of integral means of subharmonic functions and the convexity of the variations of harmonir functions over certain level curves of some other harmonic functions in 'se plane.
\end{abstract}

On an annulus, the mean value of a subharmonic function over circles of radius $r$ is a convex function of $\log r$. The purpose of the present note is to extend this result to domains of arbitrary connectivity. The main problem is to devise a suitable counterpart for $\log r$, and this is dealt with by observing that the harmonic measure of either boundary circle of an annulus is a linear function of $\log r$. In the case of a general domain $G$, our scheme is to start with a harmonic function $m$ on $G$ and form as analogues of the integral means the integrals with respect to the conjugate $m^{*}$ of $m$ over certain level curves of $m$. This results in a generalization of the classical convexity property of the integral means of a subharmonic function, comprising our main theorem.

In what follows, $G$ is a domain in the plane, $m$ is a harmonic function on $G$ such that $\{\varepsilon \leqslant m \leqslant 1-\varepsilon\}$ is compact whenever $0<\varepsilon \leqslant \frac{1}{2}$ and $m^{*}$ denotes the (multiple-valued) harmonic conjugate of $m$. Further $l_{\alpha}$ denotes the oriented level curves $m=\alpha(0<\alpha<1)$ on which $m^{*}$ increases except at the zeros of the gradient of $m$, and $V_{u}(\alpha)$ denotes the sum of total variations of a given harmonic function $u$ over the components of $l_{\alpha}$. We remark that $m$ may take values outside $(0,1)$ and our choice of the numbers 0 and 1 is for simplicity.

THEOREM 1. Let $u$ be a harmonic function on $\{0<m<1\}$ and $v$ a subharmonic function on $\{0<m<1\}$, and denote by $u^{*}$ the harmonic conjugate of $u$. Then, for $0<\alpha<1$,

(1) $\int_{l_{\alpha}} d u^{*}$ is independent of $\alpha$;

(2) $\int_{l_{\alpha}} u d m^{*}=A \alpha+B$, where $A$ and $B$ are constants, with $A$ given by the integral in (1);

(3) $\int_{l_{\alpha}} v d m^{*}$ is a convex function of $\alpha$;

Received by the editors November 25, 1974 and, in revised form, November 24, 1975.

AMS (MOS) subject classifications (1970). Primary 31A05; Secondary 30A04.

Key words and phrases. Harmonic, subharmonic, convexity, integral mean, variation, conformal mapping.

'The author wishes to thank Professor Maurice Heins for suggesting the problem and Professor Maynard Arsove for helpful comments. 
(4) $V_{u}(\alpha)$ is continuous;

(5) $V_{u}(\alpha)$ is convex over intervals of values of $\alpha$ such that $l_{\alpha}$ does not pass through zeros of the gradient of $m$.

In this, our main theorem, it should be observed that the integral in (3) actually represents a sort of mean-value of the subharmonic function $v$ over $l_{\alpha}$, in view of the fact that the integral of $d m^{*}$ over $l_{\alpha}$ is constant, by (1). We remark also that $V_{u}(\alpha)$ is not convex over $0<\alpha<1$, in general, as we shall later show by example. The remainder of the paper is devoted primarily to proving Theorem 1, and the key to the proof lies in the fact that the level lines $l_{\alpha}$ subdivide $\{0<m<1\}$ into doubly connected regions, which can then by analyzed by conformal mappings onto annuli.

\section{The case of doubly connected domains with piecewise analytic boundary curves.}

Lemma 1. Suppose $G=\{R<|z|<1\}, 0<R<1$, and $u$ is harmonic on $G$ and continuous on $|z|=1$. Then the variation of $u$ on $|z|=r$ tends to the variation of $u$ on $|z|=1$ as $r \rightarrow 1$.

Proof. We use $V(r)$ to denote the variation of $u$ on $|z|=r$.

Writing $V(1)$ as the limit of suitable sums and noting that $u\left(r e^{i \theta}\right) \rightarrow u\left(e^{i \theta}\right)$ uniformly as $r \rightarrow 1$, we have $\lim _{\inf _{r \rightarrow 1}} V(r) \geqslant V(1)$.

We shall show $\lim \sup _{r \rightarrow 1} V(r) \leqslant V(1)$. Let $R<R_{0}<r<1$ and $\varepsilon>0$. We note that $u$ is of bounded variation on $|z|=R_{0}$. Fix $f, R_{0}<r<1$. There exist $\theta_{1}<\theta_{2}<\cdots<\theta_{n}<\theta_{n+1}=\theta_{1}+2 \pi$ such that

$$
V(r)-\varepsilon<\sum_{j=1}^{n}\left|u\left(r e^{i \theta_{j+1}}\right)-u\left(r e^{i \theta_{j}}\right)\right| \equiv w(r) .
$$

Consider the function

$$
w(z)=\sum_{j=1}^{n}\left|u\left(z e^{i \theta_{j+1}}\right)-u\left(z e^{i \theta_{j}}\right)\right|
$$

on $R_{0} \leqslant|z| \leqslant 1 ; w(z)$ is subharmonic on $R_{0}<|z|<1$ and continuous on $R_{0} \leqslant|z| \leqslant 1$. Let $M(r)$ be the maximum of $w(z)$ on $|z|=r$. By Hadamard's three-circle theorem we have

$$
M(r) \leqslant \frac{\log (1 / r)}{\log \left(1 / R_{0}\right)} M\left(r_{0}\right)+\frac{\log \left(r / R_{0}\right)}{\log \left(1 / R_{0}\right)} M(1) .
$$

Since $\varepsilon$ is arbitrary, we see from (1) that

$$
V(r) \leqslant \frac{\log (1 / r)}{\log \left(1 / R_{0}\right)} V\left(R_{0}\right)+\frac{\log \left(r / R_{0}\right)}{\log \left(1 / R_{0}\right)} V(1) .
$$

Thus $\lim \sup _{r \rightarrow 1} V(r) \leqslant V(1)$. (The construction of $w(z)$ is adapted from Bieberbach and Csillag; see Beckenbach [1, p. 616].)

Lemma 2. Let $G=\{R<|z|<1\}, 0<R<1$ and $m$ be the harmonic measure of either boundary circle of $G$. Suppose $u, u^{*}$ and $v$ are as in Theorem 
1 ; moreover, $u$ and $u^{*}$ can be extended continuously to $|z|=1$, $v$ can be extended upper semicontinuously to $|z|=1$. Then whenever $l_{\alpha} \subseteq\{R<|z| \leqslant 1\}$ conclusions (1) to (5) in Theorem 1 hold.

Proof. It is clear that $m(z)=a \log |z|+b, m^{*}(z)=a \cdot \arg z+c$ for some real constants $a, b$ and $c ; l_{\alpha}$ is a circle centered at 0 with radius, say, $r(\alpha)$. We note that $\alpha=a \log r(\alpha)+b$. By the orientation of $l_{\alpha}$ we know that

$$
\int_{l_{\alpha}} d m^{*}=2 \pi|a|
$$

In $G$,

$$
u(z)=d \log |z|+\operatorname{Re} f(z) \text { and } u^{*}(z)=d \arg z+\operatorname{Im} f(z)
$$

for a single-valued analytic function $f(z)$ on $G$ and a real constant $d$. Because $u$ and $u^{*}$ can be extended continuously to $|z|=1, f(z)$ can also be extended continuously to $|z|=1$. Hence the expressions for $u$ and $u^{*}$ in $G$ are also valid for $|z|=1$. Thus, for $l_{\alpha} \subseteq\{R<|z| \leqslant 1\}$,

$$
\int_{l_{\alpha}} d u^{*}=\int_{|z|=r(\alpha)} d u^{*} \cdot \operatorname{sign}(a)=2 \pi d \cdot \operatorname{sign}(a) .
$$

From the above equality, the fact $\alpha=a \log r(\alpha)+b$ and

$$
\int_{l_{\alpha}} u d m^{*}=2 \pi|a|[d \log r(\alpha)+\text { constant }],
$$

we conclude (2).

For $R<r(\alpha) \leqslant 1, \int_{|z|=r(\alpha)} v d \theta$ is a convex function of $\log r(\alpha)$ and $\alpha$ is a linear function of $\log r(\alpha)$, hence $\int_{l_{\alpha}} v d m^{*}$ is a convex function of $\alpha$.

Continuity of $V_{u}(\alpha)$ as a function of $\alpha$ for $l_{\alpha} \subseteq\{R<|z| \leqslant 1\}$ follows from Lemma 1.

$$
V_{u}(\alpha)=\int_{|z|=r(\alpha)}\left|\frac{\partial u}{\partial \theta}\right| d \theta=\frac{1}{|a|} \int_{l_{\alpha}}\left|\frac{\partial u}{\partial \theta}\right| d m^{*}
$$

is a convex function of $\alpha$, because $|\partial u / \partial \theta|$ is subharmonic in $\{R<|z|<1\}$.

Corollary. Suppose $G$ is a doubly connected domain with piecewise analytic boundary curves and every interior point of the analytic arc represents only one accessible point on the boundary of $G$. Suppose $u, u^{*}$ and $v$ are as in Theorem 1 and $u$ and $u^{*}$ can be extended continuously, $v$ can be extended semicontinuously to a boundary component of $G$. Then for $l_{\alpha}$ in $G$ or in that boundary component, conclusions (1) to (5) in Theorem 1 remain true.

Proof. Because $G$ is doubly connected with no point-like boundary component, there exists a conformal mapping $f$ from a suitably chosen annulus $A(R, 1)$ to $G$. See Goluzin [2, p. 208] for the proof of the above fact. For the following discussion of correspondence of boundaries under conformal mapping, the reader is referred to $\$ 3$, Chapter II of Goluzin [2]. Since all the points on $\partial G$ are accessible and every interior point of the analytic arcs on $\partial G$ represents only one accessible point, $f$ admits a continuous extension to 
$\{|z|=1\} \cup\{|z|=R\}$, one-to-one except possibly at the pre-images of the endpoints of the analytic arcs.

Because harmonicity, subharmonicity, total variation and topological properties are preserved under $f$, we conclude this corollary by Lemma 2 .

2. Proof of the main theorem. Let $f=m+i m^{*}, g=f^{\prime}=m_{x}-i m_{y}, Z=$ \{zeros of $g$ in $G\}$ (i.e. the zeros of the gradient of $m$ ) and $S=\{m(z)$ : $z \in Z$ \}. Because $g$ has finitely many zeros on each compact subset of $G, S$ is countable and has no limit point in $(0,1)$. Let $a, b$ be two numbers, $0<a<b<1$ and $(a, b) \cap S=\varnothing$, and $E$ denote the set $\{a<m<b\}$.

We observe that $l_{\alpha}(0<\alpha<1)$ is compact. If $z_{0}$ is on $l_{\alpha}$ and $n$ is the multiplicity of the zero of $f-f\left(z_{0}\right)$ at $z_{0}$ then in a neighborhood of $z_{0}, l_{\alpha}$ is composed of $2 n$ analytic rays from $z_{0}$, disjoint except at $z_{0}$. Therefore $l_{\alpha}$ has finitely many components and each component of $l_{\alpha}$ is either a closed analytic Jordan curve or a closed curve composed of analytic Jordan arcs with end points in $Z$.

We claim that $E$ is a finite disjoint union of doubly connected domains. We first consider the case $a$ and $b$ are not in $S$. Because $l_{a} \cup l_{b}$ is the boundary of $E$ and $l_{a} \cup l_{b}$ is disjoint from $Z, E$ has finitely many components and each component is a domain of finite connectivity. Let $D$ be any component of $E, \partial D$ be the boundary of $D$ being oriented so that $D$ is to the left of $\partial D$ and $n$ be the number of components of $\partial D$. We want to show $n=2$. We note that $n>1$ from the maximum and minimum principle. Since $m$ is constant on each component of $\partial D$, we have $m_{x} d x+m_{y} d y=0$ on $\partial D$. Because $g$ has no zero in $D$, with the aid of the argument principle we have

$$
\begin{aligned}
0 & =\int_{\partial D} \frac{g^{\prime}}{g} d z=\text { increment of } \arg g \text { along } \partial D \\
& =\text { increment of } \arctan \left(-m_{y} / m_{x}\right) \text { along } \partial D \\
& =\text { increment of } \arctan (d x / d y) \text { along } \partial D \\
& =-2 \pi+2 \pi(n-1) .
\end{aligned}
$$

Therefore $n=2$ and $D$ is doubly connected. In case one or both of $a, b$ is in $S$, we may conclude that $E$ is a finite union of doubly connected domains by observing $E=\cup_{n}\left\{a_{n}<m<b_{n}\right\}$ if $a_{n} \downarrow a$ and $b_{n} \uparrow b$.

If $D$ is a component of $E$, from the maximum and minimum principle and the local structure of the level curves, we see that each point on $\partial D \backslash Z$ represents only one accessible point on $\partial D$. If $D^{\prime}$ is another component of $E$, we observe that $\partial D \cap \partial D^{\prime}$ is a finite set; this follows from the fact that every point in the intersection has to be a point in $Z$.

By applying the Corollary of Lemma 2 to each component of $E$ and noting that the boundary of a component of $E$ meets the boundary of some other component of $E$ at most finitely many points, we conclude that (1) to (5) are true for $\alpha \in[a, b]$. Recall that $S$ has no limit point in $(0,1)$ and let $a$ and $b$ vary, we can easily see that (1), (2), (4) and (5) are true for $\alpha \in(0,1)$. 
We shall prove $\int_{l_{\alpha}} v d m^{*}$ is convex across points in $S$. Fix $s \in S$ and choose $c, d$ so that $c<s<d$ and $(c, d) \cap S=\{s\}$. Let $U$ be the region $\{c<m<$ $d\}$; the Dirichlet problem is solvable on $U$. After approximating $v$ on the boundary of $U$ by a decreasing sequence of continuous functions and solving the Dirichlet problem for this sequence of functions, we may apply (2) and the harmonic majorant property of subharmonic functions to obtain the convexity of $\int_{l_{\alpha}} v d m^{*}$ at $s$.

The proof of Theorem 1 is complete.

3. An example. We shall show by example that $V_{u}(\alpha)$ need not be convex across points in $S$.

Let $m(z)$ be $\log \left|z^{2}-1\right|$ in $C \backslash\{1,-1\}, m^{*}$ be its conjugate and $G=\{-1$ $<m(z)<1\}$. From the Cauchy-Riemann equations, $\left(m+i m^{*}\right)^{\prime}\left(z_{0}\right)=0$ if and only if $m_{x}\left(z_{0}\right)=m_{y}\left(z_{0}\right)=0$. By a direct computation we see that 0 is the only point at which $\left(m+i m^{*}\right)^{\prime}$ vanishes. Thus $Z=\{0\}$ and also $S=\{0\}$. For a value $\alpha$,

$$
l_{\alpha}=\left\{x+i y:\left[(x+1)^{2}+y^{2}\right]\left[(x-1)^{2}+y^{2}\right]=e^{2 \alpha}\right\} .
$$

We note that (a) $l_{\alpha}$ meets the $x$-axis at $\pm \sqrt{1+e^{\alpha}}$ when $\alpha>0$, at $\pm \sqrt{1 \pm e^{\alpha}}$ when $\alpha \leqslant 0$, (b) $l_{\alpha}$ meets the $y$-axis at $\pm i \sqrt{e^{\alpha}-1}$ when $\alpha \geqslant 0$, does not meet $y$-axis when $\alpha<0$, and (c) $l_{\alpha}$ meets the unit circle at $\pm\left(\sqrt{4-e^{2 \alpha}} / 2 \pm i e^{\alpha} / 2\right)$ when $\alpha \leqslant \log 2$ and does not meet unit circle when $\alpha>\log 2$. On $l_{\alpha}$ we also have

$$
\frac{d x}{d y}=\frac{-y\left(x^{2}+y^{2}+1\right)}{x\left(x^{2}+y^{2}-1\right)} \text { and } \frac{d y}{d x}=\frac{-x\left(x^{2}+y^{2}-1\right)}{y\left(x^{2}+y^{2}+1\right)} .
$$

From the properties of $l_{\alpha}$ discussed in the last paragraph, we see that $l_{\alpha}$ is symmetric about the $x$-axis and the $y$-axis. Moreover, (A) for $0<\alpha \leqslant \log 2$, $l_{\alpha}$ is a simple closed analytic curve, on which $|y|$ increases from $\sqrt{e^{\alpha}-1}$ to $e^{\alpha} / 2$ when $x$ increases from 0 to $\sqrt{4-e^{2 \alpha}} / 2,|y|$ decreases from $e^{\alpha} / 2$ to 0 when $x$ increases from $\sqrt{4-e^{2 \alpha}} / 2$ to $\sqrt{1+e^{\alpha}}$, (B) for $\alpha=0, l_{\alpha}$ is a union of two simple closed curves analytic at every point except 0 , on which $|y|$ increases from 0 to $\frac{1}{2}$ when $x$ increases from 0 to $\sqrt{3} / 2$, and $|y|$ decreases from $1 / 2$ to 0 when $x$ increases from $\sqrt{3} / 2$ to $\sqrt{2}$ and (C) for $\alpha<0, l_{\alpha}$ is a union of two disjoint simple closed analytic curves, on which $|y|$ increases from 0 to $e^{\alpha} / 2$ when $x$ increases from $\sqrt{1-e^{\alpha}}$ to $\sqrt{4-e^{2 \alpha}} / 2$, and $|y|$ decreases from $e^{\alpha} / 2$ to 0 when $x$ increases from $\sqrt{4-e^{2 \alpha}} / 2$ to $\sqrt{1+e^{\alpha}}$.

Let $u(z)=\operatorname{Re} z=x$. Then

$$
\begin{aligned}
V_{u}(\alpha) & =4 \sqrt{1+e^{\alpha}} \quad \text { when } \alpha>0, \\
& =4 \sqrt{2} \quad \text { when } \alpha=0, \\
& =4\left(\sqrt{1+e^{\alpha}}-\sqrt{1-e^{\alpha}}\right) \quad \text { when } \alpha<0 .
\end{aligned}
$$

$V_{u}(\alpha)$ is not convex at $\alpha=0$ because it has left-hand derivative $+\infty$, right-hand derivative $\sqrt{2}$ at $\alpha=0$. This example tells us that Theorem 1(5) 
cannot be improved. We note that $V_{u}(\alpha)$ is increasing near $\alpha=0$ in this example.

We observe that $u^{*}(z)=\operatorname{Im} z=y$ and

$$
\begin{aligned}
V_{u^{*}}(\alpha) & =4\left(e^{\alpha}-\sqrt{e^{\alpha}-1}\right) \quad \text { when } 0<\alpha \leqslant \log 2 \\
& =4 \quad \text { when } \alpha=0 \\
& =4 e^{\alpha} \quad \text { when } \alpha<0
\end{aligned}
$$

$V_{u^{*}}(\alpha)$ has left-hand derivative 4 , right-hand derivative $-\infty$ at $\alpha=0$, so convexity is impossible and in fact it attains a strict local maximum at $\alpha=0$.

\section{REFERENCES}

1. E. F. Beckenbach, The stronger form of Cauchy's integral theorem, Bull. Amer. Math. Soc. 49 (1943), 615-618. MR 5, 35.

2. G. M. Goluzin, Geometric theory of functions of a complex variable, "Nauka", Moscow, 1966; English transl., Transl. Math. Monographs, vol. 26, Amer. Math. Soc., Providence, R. I., 1969. MR 36 \#2793; 40 \#308.

Department of Mathematics, University of Illinois, Urbana, Illinois 61801

Current address: Department of Mathematics, Indiana University, Bloomington, Indiana 47401 